\title{
Civilisations
}

Revue internationale d'anthropologie et de sciences

humaines

42-2 | 1993

enQuete d'identité

\section{L'identité administrée}

L'État multiethnique entre revendications et bureaucratie : le cas de la R.S.F. de Yougoslavie

\section{Claude Girard}

\section{OpenEdition}

\section{Journals}

Édition électronique

URL : http://journals.openedition.org/civilisations/2404

DOI : 10.4000/civilisations. 2404

ISSN : 2032-0442

\section{Éditeur}

Institut de sociologie de l'Université Libre de Bruxelles

\section{Édition imprimée}

Date de publication : 1 décembre 1993

Pagination : 255-270

ISBN : 2-87263-108-9

ISSN : 0009-8140

Référence électronique

Claude Girard, «L'identité administrée », Civilisations [En ligne], 42-2 | 1993, mis en ligne le 01 décembre 1996, consulté le 01 mai 2019. URL : http://journals.openedition.org/civilisations/2404 DOI : 10.4000/civilisations.2404

Ce document a été généré automatiquement le 1 mai 2019.

(c) Tous droits réservés 


\title{
L'identité administrée
}

\author{
L'État multiethnique entre revendications et bureaucratie : le cas de la \\ R.S.F. de Yougoslavie
}

\section{Claude Girard}

1 Depuis 1989, les tribulations que traverse l'Europe centrale et orientale, au premier rang desquelles figure la dramatique dislocation yougoslave, sont très fréquemment interprétées en termes de résurgences de nationalismes identitaires plus ou moins irrationnels et ataviques. A la faveur des multiples crises qui affectent pratiquement tous les pays qui se dégagent de l'épisode communiste, le phénomène identitaire semble s'imposer comme la clé de compréhension de cette fin de siècle. avec d'autant plus de violence qu'il aurait été réprimé ou refoulé pendant quarante ans. Les politiques et les diplomates consacrent désormais leur énergie à imaginer de nouveaux dispositifs de coexistence et redécouvrent le problème des minorités, tandis que l'homme de la rue n'ignore plus rien des moeurs et des revendications de peuples, dont tout, parfois jusqu'au nom même, lui était encore inconnu hier.

2 A chaud ici, plus posément là-bas, le problème des identités nationales et ethniques se retrouve posé dans l'ensemble de l'Europe ex-communiste, et même ailleurs, avec autant sinon davantage d'acuité qu'à l'issue de la Première Guerre mondiale. D'où la nécessité de tenter d'introduire un peu d'ordre dans la réflexion. Face à un écheveau aussi emmêlé, la tâche du politiste consiste sans doute à décrire et à apprécier les tenants et les aboutissants de telle ou telle crise locale, mais aussi, probablement davantage, à dénouer le contexte général dans lequel ces crises surviennent, à remettre à leur place les dimensions ethniques, historiques, géographiques, linguistiques et politiques de chaque conflit, de manière à ce que ces composantes s'éclairent les unes les autres.

3 La présente contribution ne se donnera pas une aussi vaste ambition. Elle tentera avant tout d'éclairer une dimension, peut-être sous-évaluée, de la question identitaire, qui pourrait se formuler ainsi: selon quelles logiques propres les appareils d'État sont-ils amenés à produire leurs propres discours sur l'identité et l'altérité à l'intérieur de leurs frontières territoriales? Quelles relations ces discours administratifs entretiennent-ils avec les populations concernées? Dans quelle mesure un discours objectif, dégagé de 
virtualités d'action politique, est-il possible quant à l'identité ethnique ou nationale? Dans cette perspective heuristique, « la structuration de l'identité épouse la structuration des systèmes de pouvoir ", selon la formule si nette de Georges Corm (1989: 54), au sein d'espaces pulsatiles d'échanges et d'organisation politique (Amselle, 1985).

4 Ces questions seront abordées à partir de l'exemple de la Yougoslavie, État multinational fondé en 1918, démembré en 1941 puis reconstitué par Tito qui lui donnera le nom de République socialiste fédérative de Yougoslavie (R.S.F.Y.). En mai 1991, ce pays a cessé d'exister pour exploser en une pluralité d'Etats dont les modalités de coexistence sont pour l'heure principalement guerrières. Toutefois. les questions posées par la crise de la fédération yougoslave, "poudrière " dont les barils n'ont pas encore tous explosé, se retrouvent sous une forme atténuée dans toute la région, et les termes du débat y sont probablement, mutatis mutandis, homologues, même pour des Etats beaucoup plus homogènes. Une réflexion comparatiste fouillée s'avérerait donc nécessaire, au moins dans un premier temps dans l'ensemble de l'aire culturelle balkanique.

Fondé sur une base monarchique et unitaire, reconstitué sur une base communiste et fédérale. l'Etat yougoslave constitue un cas particulièrement riche du point de vue de la production administrative de discours sur l'ethnicité. Sa disparition permet de l'étudier à l'intérieur d'une séquence historique close. Après quelques rappels historiques. nous examinerons la manière dont la R.S.F.Y. a tenté d'aménager les contradictions entre le projet yougoslave et la diversité ethnique de ses habitants, examen dont nous tirerons les enseignements pour les appliquer au contexte de la crise actuelle.

\section{Préambule : de l'âge des empires aux vicissitudes du projet yougoslave}

6 L'histoire des modes de domination politique sur le territoire ex-yougoslave se caractérise brièvement par une série d'étapes. Après la période des "grandes invasions ", le territoire est d'emblée partagé par le schisme de la chrétienté de 1054, selon une fracture qui recoupe grossièrement celle opérée dans l'Empire romain par Théodose en 395. Apparaissent simultanément plusieurs Etats dynastiques, aux limites imprécises et fluctuantes (Bozic e.a. : cartes pp. 31, 34, 136), perpétuellement en butte aux ambitions de leurs voisins, et dont les apogées successifs ont laissé leur marque dans la mémoire collective : Croatie du roi Tomislav (916-928), Rascie, puis «Empire des Serbes et des Grecs» d'Etienne IX Dusan (1331-1355), Bosnie de Tvrtko (1371-1399), etc. Seule la Slovénie, incorporée à la sphère d'influence germanique dès l'époque carolingienne, fait exception.

7 Ces Etats sont progressivement subjugués, au cours du moyen âge, par deux Empires conquérants installés au nord et au sud de l'espace yougoslave: Autriche et Empire ottoman. En dépit des nombreuses différences qui les opposent, ces Empires ont plusieurs traits communs. D'abord, ils apportent la stabilité dans la région. Les guerres entre eux n'amènent pas de renversement de l'ordre politique, mais se limitent à des réaménagements des frontières, qui seront d'ailleurs momentanément stabilisées au $17^{\mathrm{e}}$ siècle. De plus, ils représentent chacun à leur manière une forme d'universalité religieuse, ce qui les rend particulièrement indifférents aux particularismes ethniques tandis que les Habsbourgs revendiquent l'héritage de Rome à travers le Saint Empire, les Ottomans organisent la diversité de leur Empire en milletler à fondement religieux, réorientant à 
leur profit les structures politico-religieuses de Byzance dont ils sont moins les destructeurs que les successeurs.

Ce contexte est donc globalement très unificateur et n'attribue au facteur ethnique qu'une valeur marginale, presque exclusivement culturel, jamais politique. Les modes d'identification sont à cette époque à la fois étroitement localisés sur le "pays » (zupa dans l'espace yougoslave) et liés à une allégeance à une religion et à un souverain. De surcroit, les dévastations guerrières, les déplacements de la frontière interimpériale, les actions de répression contre les révoltes et les politiques de colonisation menées par les gouvernements sur leurs régions périphériques remodèlent la composition ethnique en la complexifiant. Exodes «métanastasiques » (Cvijic, 1918: 114sq.) et installation de colons allogènes aboutissent à l'enchevêtrement des ethnies et à la constitution de mémoires communautaires articulant plusieurs territoires, ce qui légitime en retour le régime impérial comme point de convergence de la diversité.

9 Le déclin de cette formule politique, à partir du $18^{\text {è }}$ siècle, sous l'effet de la montée en puissance des Etats-nations capitalistes d'Europe occidentale, et le retentissement de la Révolution française et des guerres napoléoniennes favorisent l'émergence d'une nouvelle ressource politique mobilisable pour l'émancipation périphérique: la nation moderne. Dans les Balkans ottomans toutefois, la greffe s'avère relativement artificielle, les ethnonymes ayant fini par désigner surtout l'état social: marchand (Grec ou Arménien), berger ou sériciculteur (Valaque), serf (Bulgare), pope (Serbe), (Ancel, 1992) mais aussi courrier (Tatar), musicien ambulant (Tsigane) ... Le terme «Turc» est revendiqué par les Slaves de confession musulmane, conformément à la division de l'Empire en milletler.

10 Ces obstacles n'empêchent pas les Etats qui apparaissent à la faveur du recul territorial de la Porte au $19^{\mathrm{e}}$ siècle (Serbie, autonome en 1812 ; Monténégro, qui n'avait jamais été vraiment tout à fait soumis mais dont l'indépendance est reconnue de jure) de renouer symboliquement avec les monarchies médiévales, accréditant la permanence fictive d'un sujet collectif indissolublement culturel, national et étatique. Le même phénomène se produit en Croatie demeurée vassale de Vienne, à travers un personnage comme Ante Starcevic, qui fonde le nationalisme moderne croate. Toutefois, et c'est l'originalité de l'espace yougoslave, les revendications nationalitaires s'expriment alors plutôt dans le cadre pluraliste de l'ensemble des Slaves du Sud, à travers un yougoslavisme dont la vocation est de regrouper en un seul Etat Serbes, Croates, Slovènes et, éventuellement, Bulgares. Le point d'orgue de cette ambition est l'invention d'une langue moderne commune serbo-croate, en 1850.

11 Tout est alors en place pour mettre à jour ce qui sera désormais la contradiction majeure de la vie politique yougoslave: la coexistence d'un nationalisme collectif yougoslaviste, fondé sur l'existence "objective» d'une communauté culturelle et raciale (dans le langage du $19^{\mathrm{è}}$ siècle), et de nationalismes particularistes, principalement serbe et croate, fondés sur l'existence ou le souvenir d'Etats qui eurent chacun leur heure de gloire. En 1918, l'installation sur les ruines des Empires d'un Royaume des Serbes, Croates et Slovènes, qui prendra en 1929 le nom de Yougoslavie, se révèle vite un quiproquo : le nouvel Etat apparaît avant tout comme une extension territoriale de la Serbie et le yougoslavisme est détourné en un projet exclusiviste d'unification culturelle autour des seules valeurs serbes.

12 Cette contradiction, impossible à surmonter dans le cadre sans cesse plus autoritaire de la monarchie, amène en pratique à une ethnification de la vie politique, oblitérant 
l'émergence d'une culture politique commune. Tandis que les statistiques démographiques comptabilisent une catégorie serbo-croate unique. d'après le critère linguistique, ce qui suppose le principal problème résolu (Castellan, 1991: 410), la vie parlementaire est dominée par l'affrontement dramatique entre les anciens notables serbes et le bloc des Precani, qui regroupe les leaders des anciennes provinces austrohongroises (ibid.: 413). La réconciliation sur le tard entre Serbes et Croates, par le compromis de 1939, n'empêche pas l'éclatement sanglant de l'Etat à la faveur de la Seconde Guerre mondiale. Les communistes, qui se retrouvent maîtres du pays en 1945, devront le reconstruire en réactivant l'idéal yougoslaviste et en donnant des gages aux nombreuses minorités ethniques.

Ils produiront pour cela un discours sur l'ethnicité extrêmement touffu, tout en mettant en place des structures fédérales. L'aménagement de la diversité ethnique par le nouveau pouvoir prendra essentiellement deux formes, outre bien sûr la rhétorique attendue de la construction du socialisme, qui permet là comme ailleurs d'occulter les différences présentes en les projetant dans l'avenir: un cadre territorial morcelé et une reconnaissance juridique des différentes composantes ethniques. Le premier doit être analysé comme un discours géopolitique. Le second, qui constitue le discours ethnologique de l'Etat proprement dit, appelle une analyse plus sociolinguistique ou sémiotique.

\section{Le morcellement territorial comme discours d'Etat}

Alors que la monarchie yougoslave s'était bornée à reconnaître seulement trois nations constitutives en 1918, tout en instaurant une séparation nette entre les citoyens slaves et les autres (n'accordant jusqu'en 1926 le droit de vote qu'aux premiers), l'Etat titiste est fondé sur la base de six Républiques, ce qui intègre à la fois la diversité des trois nations et la reconnaissance d'autres groupes nationaux. Toutefois, contrairement à l'U.R.S.S. par exemple, le fédéralisme yougoslave ne s'appuie pas exclusivement sur des Etats membres affectés terme à terme à des nations constitutives. Si les vieux Etats plus ou moins nationaux (Serbie, Croatie) sont restaurés, si les Slovènes, reconnus de longue date comme nation, disposent désormais et pour la première fois dans l'histoire d'une République, les cas de la Bosnie-Herzégovine, de la Macédoine et du Monténégro sont, chacun à leur manière, atypiques.

La Bosnie-Herzégovine, simple région historique promue République, représente un cas singulier. Peuplée notamment de Serbes et de Croates, mais aussi de Slaves islamisés depuis l'époque ottomane, que les nationalistes voisins revendiquent concurremment comme partie de leur peuple, elle constitue pour le pouvoir une manière de diviser les deux grandes nations sans se risquer à tracer une frontière entre elles, tout en ménageant un cadre d'expression aux Slaves musulmans. jusque là totalement marginalisés sur la scène politique. En rehaussant ceux-là, elle permet d'amoindrir l'influence numérique de ceux-ci. Pour autant, la Bosnie-Herzégovine n'est pas une entité artificielle, car ses délimitations se sont stabilisées les premières dans la constitution des frontières de l'espace yougoslave.

16 Si le Monténégro, Etat historique qui s'était de lui-même fondu dans l'ensemble yougoslave en 1918, est restauré en l'absence de toute revendication en ce sens, et les Monténégrins proclamés nation par la même occasion, c'est au nom de la même logique de division des grandes nations, ici aux dépens exclusifs des Serbes. La question de savoir 
si la distinction entre une nation serbe et une monténégrine est fondée a fait l'objet au Monténégro de débats qui se poursuivent jusqu'à aujourd'hui (Lutard, 1992b). La spécificité de cette région tient surtout à son organisation sociale, qui est longtemps restée tribale (Kuci, Niksici, Njegos, ... ) et à l'originalité des institutions politiques qu'elle a connu. La mentalité a évolué en entremêlant tradition patriarcale et idéologie marxiste, l'éthique traditionnelle de solidarité s'exerçant désormais à l'échelle nationale monténégrine et non plus seulement à celle du lignage ou de la tribu (Lutard, 1991a : 72).

Le cas macédonien est plus complexe. Territoire conquis par la Serbie lors de la Première Guerre balkanique (1912), correspondant à un tiers seulement de la Macédoine historique, cette région, à la bigarrure ethnique proverbiale, faisait l'objet depuis cette date d'un irrédentisme bulgare relayé sur place par des soutiens. L'érection en 1946 de cette expression géographique en République remplissait un triple objectif: disjonction de cette zone des influences serbe et bulgare, garantie de son allégeance au nouveau pouvoir, nouvel affaiblissement de la puissance serbe dans la fédération. La création de cette République représentait un acte particulièrement volontariste dans la mesure où furent également proclamées à cette occasion la nation macédonienne, promue titulaire de la République, et même la langue de cette nation. qui n'avait jamais été fixée. Cas exemplaire de manipulation politique de l'identité ethnique, qui n'était pas le premier dans cette région (Yerasimos, $1991: 86$ sq.).

Outre les six Républiques, la création de deux provinces autonomes rattachées à la Serbie accorde un strapontin aux minorités nationales non slaves. Le Kosovo, principalement peuplé d'Albanais, est une région stratégique ; la Vojvodine, majoritairement serbe, est un conservatoire ethnique de par son ancienne fonction de marche autrichienne, colonisée par des ressortissants de l'ensemble de l'Empire: Hongrois. Slovaques, Roumains, Croates, Ruthènes, etc. L'agitation nationalitaire au Kosovo sera à partir de 1969 le principal vecteur par lequel les problèmes nationaux interviendront directement dans la vie politique fédérale (cf. Roux, 1992).

Le fédéralisme yougoslave est donc territorialisé à partir de principes complexes, dans lesquels la volonté de donner une assise nationale à autant de groupes que possible s'amalgame à une savante pondération de ces groupes, au détriment des Serbes, les plus nombreux, de manière à conjurer le retour de l'hégémonisme d'un groupe sur les autres. La pomme de discorde que représenterait l'invention de frontières intérieures conformes aux limites ethniques, dans un contexte marqué par le poids des guerres civiles de 1941-45 et par l'imbrication extrême des ethnies, est contournée par le recours à des frontières historiques qui neutralisent les enjeux de délimitation. Dans certains cas, ce découpage territorial apparaît en lui-même déjà créateur d'ethnicité. Au nom d'une logique pragmatique fondée sur la recherche d'équilibres constitutionnels subtils, le régime redécoupe les identités et tente d'homogénéiser des différences de taille, de conscience nationale, de superficie occupée pour se doter de nations constitutives de même poids.

\section{L'ethnicité comme objet d'un discours d'Etat}

Au-delà d'une logique territoriale, le régime forge ainsi ses propres catégories identitaires pour aborder l'ethnicité de ses citoyens. Mais plutôt que de chercher à gommer les différences, ce qui est le cas général, il tente de contrôler la diversité en l'encourageant à outrance et en l'organisant. Contrairement à la monarchie qui avait tenté d'imposer une 
référence identitaire privilégiée, l'Etat communiste pratique à l'encontre de ses citoyens la politique du divide et impera, qui lui permet d'asseoir sa légitimité sur son impartialité. Mais cette politique de « mise à niveau » des différents groupes passe prioritairement par le mode de communalisation national, ce qui freine considérablement les capacités du système à évoluer vers une dynamique nationale unique.

21 La Yougoslavie titiste se heurte finalement à un paradoxe élevée sur la condamnation des nationalistes, qu'elle combat d'ailleurs vigoureusement en les décapitant régulièrement et à tour de rôle, elle ne peut échapper aux déséquilibres ethniques qu'en suscitant de nouvelles appartenances nationales. Ainsi, après les Macédoniens et les Monténégrins, elle élabore progressivement une appartenance officielle pour ses serbo-croatophones islamisés, héritiers de la culture ottomane du potor (Kitsikis, 1991 : 58), qui ne se sentent ni serbes, ni croates. Cette catégorie de citoyens, «indéterminés » sous la monarchie, «Croates musulmans» sous la Croatie ustasa, émerge par précisions successives: comptabilisés comme "musulmans sans affiliation » en 1948, puis comme "Yougoslaves sans affiliation » en 1953, ils deviennent en 1961 « Musulmans au sens national », avec un $\mathrm{M}$ majuscule soulignant le caractère ethnique et non plus confessionnel de cette appartenance (Courbage, 1991: 665, note 30). Ce n'est qu'en 1971 que les Musulmans seront définitivement reconnus comme catégorie nationale à part entière.

Qu'importe en l'occurrence que le régime paraisse contredire sa lutte contre les manifestations religieuses en faisant de l'appartenance confessionnelle un critère de nationalité. Le cas des Musulmans appelle d'autres commentaires : le terme a été préféré par exemple à Bosniaques, référent commun dans la vie quotidienne mais laissant entendre que Serbes et Croates ne seraient pas chez eux en Bosnie. Il permet de rassembler dans une même catégorie des habitants de régions différentes (Bosnie et Sandjak principalement), sans les affaiblir en postulant implicitement une communauté d'intérêts. Enfin, s'il est évident que tous les musulmans du pays ne sont pas Musulmans, puisqu'ils peuvent appartenir à d'autres catégories nationales (la plupart des Albanais, certains Roms, les Turcs, etc.), l'inverse est également vrai : un Musulman pris individuellement peut s'avérer athée, voire membre de la Ligue des communistes !

Si l'on va au-delà de cet exemple, on conçoit que les catégories identitaires qu'a connu, au cours de l'histoire, le territoire de l'ex-Yougoslavie peuvent être regroupées au sein d'un répertoire dans lequel les discours contemporains ayant trait à la nationalité puisent de manière sélective. L'ethnonyme est alors considéré sous l'angle, non pas de son adéquation à une réalité, mais de la ressource symbolique qu'il procure dans un champ politique. Il convient d'appréhender ce thesaurus d'ethnonymes comme éléments de classifications mouvantes et tenter de déceler des logiques de classement. Cela suppose au préalable de hiérarchiser ces dénominations en fonction de l'identification particulariste, régionaliste, étatiste ou nationale qu'elles suggèrent à un moment donné, en sachant que le type de connotation peut évoluer dans le temps.

Mais si l'on s'en tient au discours administratif sur la diversité ethnique, il apparaît vite que les taxinomies se chevauchent et s'interpénètrent sans aucune autre logique que celle de la représentation pragmatique. Dans les recensements de la R.S.F.Y, le critère linguistique est certes distingué du critère ethnique, mais contribue à le fonder, tout en s'associant parfois pour cela à l'obédience religieuse. Du reste, la dialectologie de la langue serbo-croate ne renvoie pas aux catégories nationales, même si Serbes et Croates ont leur propre alphabet et produisent des normes linguistiques divergentes (Garde, 1992 : 127 sq.). En outre, les citoyens peuvent également opter pour une appartenance 
régionale, ou même s'abstenir de déclarer une appartenance. Les statistiques étatiques sont néanmoins redressées selon une logique de simplification qui aboutit à leur faire tenir le discours autonome de l'Etat sur l'identité ethnique.

La classification finale (cf. Komac, 1991: 158-159) procède de compromis. Tandis que quelques nouvelles catégories s'imposent, on constate la disparition de références autrefois courantes, comme Chopes, Morlaques, «Uscoques", Souabes, etc., sans parler des références régionales ou liées à d'anciennes structures étatiques ou administratives comme Carnioliens, Dalmates, Poglisiens, Bokezi... Il serait périlleux de conclure globalement que ces références ne s'appliquent à plus personne, une « sémio-histoire » de chacune de ces dénominations restant à écrire. Parfois, les groupes obtiennent de changer de dénomination, comme les Albanais, qui deviennent en 1967 des Albanci, terme à connotation distante et respectueuse qui remplace les Skipetari ou Siptari, termes perçus comme péjoratifs par les intéressés (Garde,1992:231). Cette bienveillance s'accompagne d'une oeuvre législative sur les minorités d'une envergure exceptionnelle, à travers laquelle les Roms (Tsiganes) par exemple, bénéficient d'une insertion sans commune mesure avec l'assimilationnisme répressif et raciste dont ils peuvent faire l'objet dans les pays voisins.

26 Toutefois, il n'y a pas de réelle «objectivité statistique »: des particularismes microcommunautaires sont validés ici, ignorés ailleurs. Ainsi par exemple, les Arbâresh de la région de Zadar sont enregistrés à part du reste des Albanais; le mode de vie sédentaire ou nomade distingue les deux rubriques des Turcs et des Yürüks; les catégories d'Ukrainiens et de Ruthènes s'opposent sur la base religieuse, orthodoxe pour les uns, gréco-catholique pour les autres ; les Autrichiens sont enregistrés à part des Allemands. A contrario, la diversité culturelle des Roms n'est pas validée; les Torbesi, Macédoniens islamisés, ne sont pas recensés à part ; en Vojvodine. les groupes serbes mais catholiques des Sokci et Bunjevci (cf. Sellier, Sellier, 1991 : 44) sont ignorés. Enfin, des dénominations d'usage populaire comme Valaques, Tsintsares, sont inégalement retraduites en catégories ethnolinguistiques d'apparence plus «sérieuse »: tandis qu'en Macédoine, Aroumains et Méglénoroumains sont soigneusement distingués, la Serbie et l'Istrie ne connaissent que des "Valaques", là où l'on s'attend à trouver respectivement des Aroumains et des Istroroumains. La bienveillance envers la richesse culturelle d'une population pluraliste n'exclut pas l'apparition de biais taxinomiques.

D'autre part, une tradition administrative consiste à recenser dans leur commune d'origine les émigrés partis gagner leur vie à l'étranger ou dans une autre partie de la Yougoslavie. L'administration qualifie tout à fait abusivement ces catégories d'émigrés "provisoires ». Ce fait méconnu entraîne une majoration de la population de certaines zones périphériques atteintes par l'exode rural. A l'inverse, il occulte les dynamiques des brassages ethniques dans les centres attractifs (grandes villes, zones prospères comme la Slovénie) (Pechoux, Roux, 1983). Dans le contexte actuel d'épluchage fiévreux des statistiques démographiques des régions contestées, il est important de savoir que des zones comme l'Herzégovine occidentale, la Posavina bosniaque, la Dalmatie, la Lika, la Slavonie centrale, le Polog ou la Pélagonie comptent depuis plusieurs recensements parmi les régions les plus dévitalisées de la fédération.

Enfin, la garantie constitutionnelle qu'ont les citoyens de choisir et de ne pas choisir leur identification leur permet de retourner comme un gant le discours ethnologique de l'Etat. La catégorie "Yougoslaves ", enregistrée pour la première fois en 1971, est le réceptacle privilégié d'opérations de camouflage. A l'origine, elle accueille pêle-mêle 
nomenklaturistes, yougoslaves par option politique ou par carrière militaire, athées, enfants issus de mariages mixtes. Plus tard, il arrive que des fractions de petits groupes minoritaires bien intégrés, comme par exemple des Tchèques en Slavonie, optent pour l'inscription dans la catégorie yougoslave, dans un contexte où la montée des nationalismes les pousse à se faire discrets.

De telles stratégies d'appropriation et de détournement de la grille identitaire de l'Etat expliquent les variations erratiques qui affectent parfois les statistiques démographiques d'un recensement sur l'autre. Les groupes les moins différenciés les uns des autres ont tendance à évoluer en compensant leurs gains et leurs pertes, la même personne pouvant se déclarer d'une année sur l'autre par exemple turque, puis musulmane ou albanaise en fonction de choix politiques conjoncturels ou d'intérêts immédiats. Les écarts statistiques entre catégorie nationale déclarée et langue maternelle déclarée permettent d'évaluer ces marges d'indétermination. La couche sociale la plus encline à jouer les caméléons démographiques, parce que la plus indifférente à la revendication identitaire (Stewart, 1991 :41), est celle des Tsiganes, qui se déclarent volontiers membre de la nationalité dominante à l'endroit où ils vivent. Les groupes socialement ou culturellement dominés se retrouvent ainsi minorés de leur propre initiative. Ce jeu de cache-cache identitaire peut aller assez loin : en 1991, des Tsiganes de Macédoine ont même obtenu le droit de se faire enregistrer comme Égyptiens ! (Bougarel, 1992 :92)

Le système d'organisation de la diversité ethnique de la R.S.F.Y. apparaît finalement comme un curieux mélange de manipulations bureaucratiques parfois hardies et de prise en compte de revendications particularistes, sur fond de modèle politique autoritaire (Girard, 1992 106). C'est un système dynamique, capable dans une certaine mesure de renégocier les équilibres initiaux. L'adéquation entre le dispositif administratif de gestion de la diversité et les revendications des individus est inégale et imparfaite, mais on imagine mal une correspondance mécanique entre les deux niveaux. Néanmoins, nous savons que ce modèle a finalement échoué. En quoi ses contradictions internes permettent-elles d'éclairer la crise actuelle?

\section{De l'identité administrée à l'identité exacerbée : quelques commentaires}

31 L'échec de la Yougoslavie à se transformer en démocratie consociétale à la suisse (juxtaposition de communautés réglant les questions relevant de l'échelon étatique par consensus) rappelle l'échec de l'Empire habsbourgeois à se transformer en «Suisse orientale». Mais il provient pour une large part de la contradiction dans laquelle la R.S.F.Y. s'est progressivement engagée en passant du modèle communiste orthodoxe à une synthèse originale d'autogestion économique et de décentralisation politique. Tout en se condamnant à placer l'ethnicité au centre du jeu politique, pour tenter de mieux en conjurer les effets délétères, l'Etat central n'est jamais apparu suffisamment transparent pour gérer la diversité ethnique avec équité.

La direction confédéraliste que le pays a prise après la mort de Tito (1980), avec l'établissement d'une présidence collégiale tournante, et la dégradation concomitante de la situation économique ont produit une réactivation des identités locales qui a presque joué exclusivement au profit de la nation. Le fait national a éclipsé tous les autres modes de communalisation, sauf, partiellement, en Bosnie, où la mobilisation s'est 
concurremment effectuée à partir de l'identification régionale. De ce point de vue, l'équivalence que l'Etat avait tenté d'instaurer entre ses différentes catégories nationales s'est avérée un échec. Sur le plan du patrimoine symbolique politiquement mobilisable, les différentes composantes de la fédération se sont retrouvées en situation de profonde inégalité.

Tout en gardant à l'esprit que les Républiques ont abordé la transition démocratique en ordre dispersé en fonction de leur propre culture politique (Krulic, 1989, 1991), on doit constater en effet l'avantage politique pour les nationalistes à disposer dans leur patrimoine historique d'un droit d'Etat. Cela vaut avant tout pour les Croates, mais les Serbes y ont puisé pour leur nouvel Etat, la République fédérale de Yougoslavie (R.F.Y.), le fondement de leur revendication du statut de légataire universel de la R.S.F.Y. Le cas slovène est différent : le droit d'Etat ne peut s'y prévaloir que du passé récent. Le rapport au territoire est problématique en ce sens qu'aucun royaume, duché, margraviat ou quoi que ce soit de ce genre n'a jamais porté le nom de Slovénie jusqu'à l'avènement de Tito. De là sans doute le juridisme sourcilleux qui a fait l'originalité du divorce slovène.

Le cas slovène démontre au demeurant que les cadres territoriaux hérités de la fédération titiste peuvent être récupérés. Les configurations territoriales à travers lesquelles se joue aujourd'hui la dislocation yougoslave recouvrent donc tantôt des Etats anciens restaurés. tantôt des Républiques consacrées par le titisme, tantôt, au pire, des conglomérats dont la cohérence est contestée de l'extérieur (Bosnie-Herzégovine, Macédoine, voire Kosovo). A cette inégalité s'ajoute l'impossibilité, pour la Bosnie-Herzégovine avant tout, à définir un projet politique autrement qu'à contre-courant des tendances nationalistes désormais un peu partout dominantes - fussent-elles réprimées, comme au Kosovo. Le conflit bosniaque, déclenché en 1992, peut se lire comme un conflit idéologique fondamental entre des Etats-nations unitaires, populistes et finalement antidémocratiques (Melchior, 1993; Samary, 1992) et un Etat dont le projet de citoyenneté transcende les particularismes culturels (Samary, 1993).

$\mathrm{Au}$ point de déroute où cette guerre a mené la Bosnie-Herzégovine, il est désormais rétrospectivement évident qu'un tel projet de citoyenneté pluraliste constituait au regard de ses conditions de réalisation une utopie. L'échec de ce projet était inscrit dans le fait que la seule force politique qui le soutenait était le Parti de l'action démocratique (SDA), pôle de regroupement électoral des Musulmans. Ce hiatus privait en outre les Musulmans de tout "noyau symbolique» autour duquel organiser leur défense (HladnikMilharcic,1993). En Bosnie aujourd'hui, les déplacements massifs et contraints de populations, principalement musulmanes (Le livre noir... 1993), mettent ce groupe devant l'urgence terrible d'une réorientation de sa stratégie: puisque la république proclamée en 1992 n'existe déjà plus dans les faits, l'heure est peut-être venue pour les Musulmans d'imaginer un cadre étatique qui leur soit propre, pour ne pas risquer une pure et simple disparition par élimination physique et/ou diasporisation hors de leur habitat historique.

La nation musulmane, qui n'a pas vingt-cinq ans, risque pour survivre de devoir constituer en vitesse un précipité historique qui fasse le poids face à ses deux puissants voisins. Il n'est donc pas impossible que l'accélération des événements conduise à la tentative de création d'une «Musulmanie » quelque part sur tout ou partie des ruines de la Bosnie. Mais peut-être est-il déjà trop tard pour cette révision à la baisse des ambitions des dirigeants musulmans : il n'est pas du tout évident que les Serbes et les Croates, enhardis par leurs succès, soient disposés à tolérer un Etat-tampon national musulman, 
fut-il plus ou moins «croupion ». Le chapitre bosniaque du démembrement yougoslave pose en définitive le problème de l'existence et du devenir d'un peuple.

Les aspects kosovar et macédonien de la crise appelleraient eux aussi des commentaires particuliers. A l'heure actuelle, cependant, la situation n'a pas encore irrémédiablement dégénéré dans ces régions mais l'atmosphère de veillée d'armes (l'expression est en partie mal choisie : les Albanais du Kosovo n'en ont pas), alourdie par les menaces de réactions en chaîne internationales, incite à la réserve. On peut toutefois remarquer qu'en Macédoine, un peu comme en Bosnie, le manque de consistance historique de l'identité nationale macédonienne - tout au moins dans le regard des voisins de ce pays, mais le soupçon dans ce cas précis n'est pas complètement illégitime, même si aujourd'hui, indubitablement. les Macédoniens revendiquent une conscience nationale propre - joue objectivement contre la stabilité du nouvel Etat.

Quoi qu'il en soit, le facteur national se révèle une arme politique puissante dans ce contexte de redistribution des cartes. Il ne préexiste pas comme assise objective de la conflictualité, mais s'élabore dans la longue durée comme instrument privilégié d'un type particulier de mode de domination. En tant qu'elle est désormais, au terme d'un long et douloureux processus historique. perçue dans les Balkans comme légitime et allant de soi, la nationalité offre à présent un support solide pour des entreprises politiques. Mais dans cette modernisation au miroir de l'Europe occidentale, il n'est pas sûr que les peuples aient gagné autre chose qu'aliénation et « hystérie politique » (Bibo, 1993 : 139). L'espace yougoslave est aujourd'hui agité par une nouvelle phase de sa balkanisation et ce sont les populations dont l'identité est encore structurée de la manière la plus riche, la plus gigogne, la plus polyphoniquement et dialectiquement syncrétique, bref la moins étroitement nationale, qui en font encore une fois le plus les frais. Produit contradictoire d'une modernité catastrophique et convulsive, le «nationalisme tribal» (Arendt, 1982: 185 sq.) y impose sa conception totalisante et intégriste de l'identité, ultime programme politique au nom duquel la destruction de l'autre est un prix acceptable et l'oppression une douce contrainte si elle est le fait de son propre Etat national.

\section{BIBLIOGRAPHIE}

Amselle (J.-L.), 1985. Ethnies et espaces : pour une anthropologie topologique, in s. dir. Amselle (J.-L.). M’Bokolo (E.), Au coeur de l'ethnie. Ethnies, tribalisme et Etat en Afrique. La Découverte. Paris : 11-48.

Ancel (J.). 1992. Peuples et nations des Balkans. Préface de P. George, Editions du C.H.T.S .. Paris, $230 \mathrm{p}$.

Arendt (H.), 1982. Les origines du totalitarisme. L'impérialisme. Traduit de l'anglais par M. Leiris. Fayard, Paris, $350 \mathrm{p}$.

Bibo (I.). 1993. Misère des petits Etats d'Europe de l'Est. Traduit du hongrois par G. Kassai. Nouvelle édition revue et corrigée. Albin Michel, Paris. 430 p.

Bougarel (X.). 1992. Bosnie-Herzégovine : anatomie d'une poudrière. Hérodote. 67 : 84-147. 
Bozic (I.), Cirkovic (S.), Ekmecic (M.), Dedijer (V.), 1973. Istorija Jugoslavije. 2è éd. Prosveta, Belgrade, VIII + $608 \mathrm{p}$.

Castellan (G.). 1991. Histoire des Balkans. XIVè-XXè siècle. Fayard. Paris. 543 p.

Corm (G.). 1989. L'Europe et l'Orient. De la balkanisation à la libanisation : histoire d'une modernité inaccomplie. La Découverte. Paris. 384 p.

Courbage (Y.), 1991. Les transitions démographiques des musulmans en Europe orientale. Population. $3: 651-678$.

Cvijic (J.). 1918. La péninsule balkanique. Géographie humaine avec trente et une cartes et croquis dans le texte et neuf cartes hors texte. Armand Colin. Paris. VIII + 532 p.

Garde (P.). 1992. Vie et mort de la Yougoslavie. Fayard, Paris, 444 p.

Girard (c.), 1992. Une « guerre tribale » en Yougoslavie? Un éclairage comparatiste entre Afrique et Balkans. Afrique 2000.9: 99-110.

Hladnik-Milharcic (E.), 1993. Musulmans de Bosnie, encore un effort pour devenir nationalistes ! Courrier international, 128 : 13 (art. paru dans Mladina, Ljubljana).

Kitsikis (D.), 1991. L’Empire ottoman. 2è éd. P.U.F., Paris, 127 p.

Komac (M.). 1991. Nationalités et minorités en Yougoslavie. L'événement européen, 16 : 155-175.

Krulic (J.J, 1989. La crise du système politique yougoslave. Revue française de science politique, 3 : 245-258.

Krulic (J.), 1991. L'année politique : démocratisation et désintégration, in s. dir. E. Lhomel, T. Schreiber, L'Europe centrale et orientale. De l'espoir aux réalités, Documentation française, Paris : 259-269 (Notes et études documentaires, 4942-43).

Le livre noir de l'ex-Yougoslavie : purification ethnique et crimes de guerre. Documents rassemblés par Le nouvel observateur et Reporters sans frontières. Préface : P. Bouchet. Arléa. Paris, 1993, $490 \mathrm{p}$.

Lutard (C.), 1992a. Le conflit national en Yougoslavie. Vingtième siècle. 33 : 65-76.

Lutard (C.), 1992b. Le Monténégro est-il une nation ? Le monde diplomatique, 459 : 3 (Reproduit en 1993 dans Manière de voir. 17 : 58-59).

Melchior (J.-P.), 1993. Du nationalisme serbe. Les temps modernes. 560 : 42-62.

Pechoux (P.-Y.). Roux (M.), 1983. Évolution de la population de la Yougoslavie 1971-1981.

Méditerranée. 50 (4) : 27-35.

Roux (M.). 1992. Les Albanais en Yougoslavie. Minorité nationale. territoire et développement. Editions de la Maison des Sciences de l'homme, Paris. 548 p.

Samary (C.), 1992. Au nom de l'effort de guerre et de la cause nationale.

La dérive d'une Croatie « ethniquement pure ». Le monde diplomatique, $461: 3$ (Reproduit en 1993 dans Manière de voir. sous le titre : Périlleuses dérives en Croatie. 17 : 54-55).

Samary (C.). 1993. Contre tout projet de partition. des Bosniaques favorables à l'Etat multiethnique. Le monde diplomatique. $467: 13$.

Sellier (A.), Sellier (J.), 1991. Atlas des peuples d'Europe centrale. Cartographie : A. Le Fur. La Découverte, Paris, 192

Stewart (M.), 1991. Un peuple sans patrie. Terrain. 17 : 39-52. 
Yerasimos (S.), 1991. Balkans : frontières d'aujourd'hui, d'hier et de demain ? Hérodote, 63 :

80-98.

\section{RÉSUMÉS}

Far from representing an objective phenomena, ethnie identity is closely submitted to political control that structurates it and forms it with time. The case of ex-Yugoslavia illustrated the creation by a state mechanism of a classificatory approach regarding identity and otherness inside the state territory. The geopolitical analysis and the examination of the ethnonymical thesaurus that can be extrated from censures show how R.S.F.Y. tried to overcome and to fight against its ethnie heterogeneity by manipulating bureaucratically identitary references. Moreover, the political dimensions of these procedures provide several facts which enable to understand the violent crisis which the Yugoslavian region is undergoing since 1991.

INDEX

Mots-clés : état, Yougoslavie, bureaucratie, identité, ethnicité

Keywords : state, Yugoslavia, bureaucracy, identity, ethnicity

\section{AUTEUR}

\section{CLAUDE GIRARD}

Université de Droit, d'Économie et des Sciences, Aix-Marseille 